
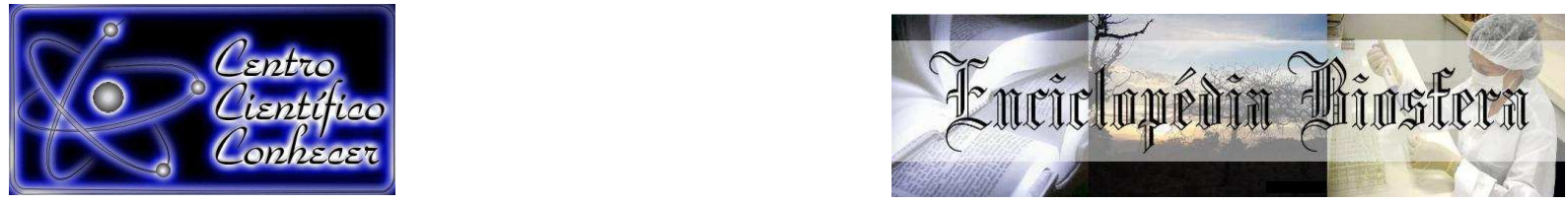

\title{
ANÁLISE DA ADESÃO AO TRATAMENTO ANTI-HIPERTENSIVO E INFLUÊNCIA DOS HÁBITOS DE VIDA
}

Rubiana Neves Ramos ${ }^{1}$, Alcione Oliveira de Souza², Edivan Rodrigues de Paula Ramos $^{3}$, Mirian Ueda Yamaguchi ${ }^{4}$

${ }^{1}$ Fisioterapeuta. Graduanda do curso de Medicina do Centro Universitário de Maringá - UniCesumar, Maringá, Paraná, Brasil.

${ }^{2}$ Enfermeira. Mestre pelo Programa de Mestrado em Promoção da Saúde do Centro Universitário de Maringá - UniCesumar, Maringá, Paraná, Brasil.

${ }^{3}$ Farmacêutico. Doutor em Ciências Biológicas pela Universidade Estadual de Maringá. Docente Adjunto II do Departamento de Medicina da Universidade Federal de Sergipe (UFS), Lagarto, Sergipe, Brasil.

${ }^{4}$ Farmacêutica. Doutora em Ciências Farmacêuticas pela Universidade Estadual de Maringá. Docente no Programa de Pós-graduação em Promoção da Saúde e

no curso de Medicina no Centro Universitário de Maringá - UniCesumar e pesquisadora do Instituto Cesumar de Ciência, Tecnologia e Inovação - ICETI, Maringá, Paraná, Brasil.

Autor Responsável: Rubiana Neves Ramos. UNICESUMAR. Maringá, Paraná, Brasil. Fone: + 5544 99937-4797. E-mail: rubsnr@hotmail.com

Recebido em: 08/04/2017 - Aprovado em: 10/06/2017 - Publicado em: 20/06/2017 DOI: 10.18677/EnciBio_2017A118

Hipertensão arterial sistêmica (HAS) é considerada um problema de saúde pública. Considerando que a adesão ao tratamento é um desafio para o controle da HAS, este estudo teve como objetivo avaliar o nível de adesão pelo Teste de Morisky-Green (TMG) e relacionar com a qualidade de vida desses indivíduos. Trata-se de estudo descritivo transversal e analítico realizado com 401 indivíduos hipertensos que são usuários do Programa "Estratégia Saúde da Família", que fazem uso rotineiro de medicação anti-hipertensiva e que participam do programa HIPERDIA. Foi utilizado questionário estruturado para coleta de dados sociodemográficos e hábitos de vida, e concomitantemente o TMG para avaliar a adesão ao tratamento medicamentoso anti-hipertensivo. Neste estudo, 59,60\% dos participantes admitiram já terem esquecido de tomar a medicação e 41,89\% desses acreditam serem descuidados quanto ao uso da medicação. Indivíduos acima do peso e que não se preocupam com um estilo de vida saudável tem menos chances de aderirem ao tratamento anti-hipertensivo. A mudança de hábitos e de estilo de vida dos hipertensos deve ser enfatizada, pois faz parte da assistência terapêutica. É fundamental dar ênfase nas condutas de tratamento não 
medicamentoso, especialmente quanto à reeducação alimentar, atividades físicas, cessação do hábito tabágico e ingesta de álcool, o que possivelmente reduziria os riscos de complicações e consequente oneração do sistema público.

PALAVRAS-CHAVE: fatores de risco, hipertensão, tratamento medicamentoso.

\title{
ANALYSIS OF ADHERENCE TO ANTI-HYPERTENSIVE TREATMENT AND INFLUENCE OF LIFE HABITS
}

\begin{abstract}
Sistemic arterial Hypertension (SAH) is considered a public health problem. Considering that treatment adherence is a challenge in controlling $S A H$, the objective of this study was assess the adherence level using the Morisky-Green Test (TMG), and correlate with the quality of life of these individuals. This descriptive, cross-sectional and analytical study was performed with 401 hypertensive individuals who are users of the "Family Health Strategy" Program; Who routinely use antihypertensive medication and who participate in the HIPERDIA program. A structured questionnaire was used to collect sociodemographic data and life habits, and concomitantly the TMG to assess adherence to antihypertensive drug treatment. In this study, $59.60 \%$ of the participants admitted that they had forgotten to take the medication and $41.89 \%$ of those believed to be careless about their medication use. Individuals who are overweight and who do not care about a healthy lifestyle are less likely to adhere to antihypertensive treatment. The change in habits and lifestyle of the hypertensive should be emphasized, as it is part of the therapeutic assistance. It is essential to emphasize non-drug treatment, especially regarding food re-education, physical activities, cessation of smoking and alcohol intake, which could reduce the risks of complications and consequent encumbrance of the public system.
\end{abstract}

KEYWORDS: drug therapy, hypertension, risk factors.

\section{INTRODUÇÃO}

A Hipertensão arterial sistêmica (HAS) é um importante desafio para a saúde pública em todo o mundo, apresentando alta frequência e morbidade. Devese assim dar maior importância à prevenção, detecção, tratamento e controle desta condição (KEARNEY, 2005 e JOHO, 2012). Em 2000, a prevalência da HAS na população mundial era de $25 \%$ e a estimativa para o ano de 2025 é que esta chegue aos 29\% (TALAEI, Et al., 2014). Para 2030 a Organização Mundial de Saúde prevê quase 23,6 milhões de mortes por doenças cardiovasculares (WORLD HEALTH ORGANIZATION, 2011). No Brasil, estudos demonstraram que a prevalência de HAS variou entre $22,3 \%$ e $44,4 \%$ dependendo dos critérios de avaliação adotados (CESARINO, 2008 e ROSÁRIO, 2009), e por ser comumente assintomática, até $50 \%$ dos indivíduos desconhecem serem hipertensos (RAMOS, 2014).

O tratamento desta afecção objetiva o controle da pressão arterial por meio de mudanças no estilo de vida associado a medidas terapêuticas apropriadas, e a baixa adesão é uma das causas de falha no tratamento anti-hipertensivo (RABBIA, 
et al., 2016), e muitas vezes os pacientes só se atentam quando as complicações já estão instaladas (LIMA, 2016). A adesão ao tratamento é influenciada por inúmeros fatores, dentre eles: idade, sexo, fatores psicológicos, falta de conhecimento relacionado à doença e ao tratamento, bem como número de medicamentos e regimes de administração destes (MORISKY, 2008, BEZERRA,2014). Já foi demonstrado também que a adesão do paciente à terapêutica está na dependência dos possíveis efeitos desta (KRONISH, 2011). Um outro fator que chama atenção é que $50 \%$ dos portadores de HAS desistem do tratamento ainda no primeiro ano (YGUSHI, 2013).

A adesão ao tratamento engloba a administração correta da medicação e mudanças no estilo vida, que incluem o controle do peso, a melhora do padrão alimentar, a redução do consumo do sal, a moderação no consumo de bebidas alcoólicas, a prática regular de atividade física, gerenciamento do estresse e a abstenção do tabagismo (DEMONER, 2012). Existem métodos que podem quantificar essa adesão, como os questionários de Haynes e Sacket e o de Morisky-Green, além da mensuração eletrônica que é um método de dispensação eletrônica, denominado pillbox (Sistema de Monitoramento de Eventos de Medicação-MEMS, Corporação Aardex, Genebra, Suíça) ${ }^{R}$ (SANTOS et al., 2013). Contudo não se chegou ao consenso sobre o melhor método para tal avaliação (BORGES, 2012). Dentre as ferramentas utilizadas o teste de Morisky-Green é considerado padrão ouro devido ao alto valor preditivo para avaliação da adesão às terapias medicamentosas (MORISKY, 1986 e VARLETA, 2015).

O objetivo deste estudo foi avaliar a adesão ao tratamento anti-hipertensivo e relacionar com fatores modificáveis da qualidade de vida desses indivíduos.

\section{MATERIAL E MÉTODOS}

Trata-se de um estudo descritivo, transversal e analítico, no qual quantificou-se previamente o número de hipertensos cadastrados na Secretaria de Saúde Municipal para que a amostra demonstrasse a realidade do município.

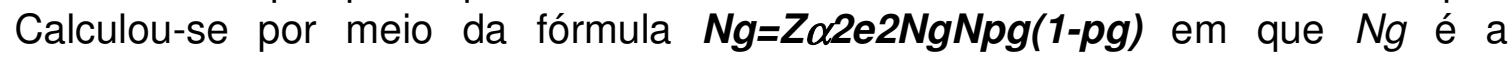
quantidade de pacientes registrados na $g$-ésima UBS, $N$ é o número total, ,pg a prevalência das características a serem pesquisadas, fixada como 0,5 para todos os grupos. Ainda, o nível de significância considerado foi de $\alpha=5 \%$ e o erro máximo admitido entre a estimativa e o valor real do parâmetro foi de $e=0,05$, isto é, de cinco pontos percentuais.

Foram entrevistados 401 indivíduos distribuídos nas 30 Unidades Básicas de Saúde (UBS) na cidade de Maringá, PR, Brasil.

Incluiu-se na pesquisa pacientes com hipertensão arterial sistêmica usuários do Programa "Estratégia Saúde da Família" do município de Maringá, PR; maiores de 18 anos; de ambos os sexos; cadastrados no mínimo há seis meses; que faziam uso rotineiro de medicação anti-hipertensiva e que participavam do programa HIPERDIA.

Foi utilizado questionário estruturado para coleta de dados sociodemográficos (sexo, idade, estado civil, escolaridade e ocupação) e informações sobre os hábitos de vida (tabagismo, alimentação, consumo de 
bebida alcoólica e atividade física). Concomitantemente foi aplicado o teste de Morisky-Green (TMG), o qual avaliou a adesão ao tratamento anti-hipertensivo.

O teste de Morisky-Green avaliou o uso inadequado de medicamentos que, segundo os autores, pode se dar das seguintes formas: esquecimento, falta de cuidado, interrupção do medicamento quando se sentir melhor ou pior. Consiste em quatro perguntas, e uma resposta afirmativa a qualquer uma dessas perguntas classifica o indivíduo como não aderente (MORISKY, 1986).

As análises estatísticas foram realizadas por meio do ambiente estatístico $R$ ( $R$ Core Team), versão 3.2.2. O nível de significância foi fixado em $5 \%$ para todos os testes. Com o intuito de investigar possíveis associações entre a adesão ao tratamento anti-hipertensivo, determinada pelo teste de Morisky-Green, com fatores relacionados a atividade física, nutrição, excesso de peso, cigarro e álcool, foram calculadas as odds ratios (razões de chances), assim como seus respectivos intervalos de confiança de $95 \%$ por meio da regressão logística univariada. Posteriormente, foram selecionadas as variáveis que apresentaram associação ao menos moderada $(p<0,25)$ com a variável que indica a aderência ao tratamento pelo teste qui-quadrado.

Este estudo foi aprovado pelo Comitê de Ética em Pesquisa do Unicesumar, parecer número 873-870 e também pelo CECAPS (Assessoria de Formação e Capacitação Permanente dos Trabalhadores de Saúde), órgão que regulamenta e aprova as pesquisas realizadas nas UBSs em Maringá. Todos os participantes assinaram o Termo de Consentimento Livre e Esclarecido, concordando em participar do estudo.

\section{RESULTADOS E DISCUSSÃO}

O último levantamento elaborado a partir dos dados do Sistema de Informação da Atenção Básica (SIAB) do Ministério da Saúde encontrou no Município de Maringá 25.672 casos de indivíduos hipertensos cadastrados, o que traduz uma prevalência de $6,4 \%$. A amostra estudada nesta pesquisa foi composta de 401 indivíduos denotando 1,56\% dos hipertensos cadastrados. De modo geral, os hipertensos avaliados neste estudo são predominantemente do gênero feminino ( $n=281$ ou $70,1 \%$ ), com idade entre 60 e 79 anos ( $n=200$ ou $50 \%$ ), primeiro grau completo ou incompleto ( $n=179$ ou $44,5 \%)$, casados ( $n=179$ ou $44,5 \%$ ), com apenas um filho ( $n=268$ ou $66,9 \%$ ), que moram com duas ou mais pessoas em sua residência ( $n=297$ ou $74,1 \%$ ) e que trabalham ( $n=372$ ou $92,8 \%$ ). Apesar de 120 pacientes serem aposentados (29,9\% dos entrevistados), apenas $29(7,2 \%)$ deles relataram não ter atividade laboral remunerada. A maioria dos pacientes apresentou valores do Índice de Massa Corporal (IMC) indicativos de sobrepeso $(n=165$ ou $41,1 \%$ ) seguidos por aqueles com valores indicativos de obesidade ( $n=140$ ou $39,9 \%$ ).

$\mathrm{Na}$ Tabela 1 encontram-se as respostas dos pacientes para o Teste de Morisky-Green, em que 59,60\% dos participantes admitiram já terem esquecido de tomar a medicação ao menos alguma vez; 41,89\% desses acreditam serem descuidados quanto ao uso da medicação. Os maiores percentuais frente a administração correta da medicação foram: não deixar de ingerir a medicação quando se sente melhor (93,52\%), nem tampouco quando se sente pior $(97,01 \%)$. 
Tabela 1: Frequência das respostas empregados na avaliação da adesão ao tratamento antihipertensivo

\begin{tabular}{|c|c|c|}
\hline Perguntas do Teste de Morisky-Green & $\mathbf{n}$ & $\%$ \\
\hline \multicolumn{3}{|l|}{ Você alguma vez se esqueceu de tomar o remédio? } \\
\hline Sim & 239 & 59,60 \\
\hline Não & 162 & 40,40 \\
\hline \multicolumn{3}{|l|}{ Você às vezes é descuidado para tomar seu remédio? } \\
\hline $\operatorname{Sim}$ & 168 & 41,89 \\
\hline Não & 233 & 58,11 \\
\hline \multicolumn{3}{|l|}{ Você às vezes é descuidado para tomar seu remédio? } \\
\hline Sim & 26 & 6,48 \\
\hline Não & 375 & 93,52 \\
\hline \multicolumn{3}{|c|}{ Quando você se sente melhor, às vezes, você para de tomar seu remédio? } \\
\hline Sim & 12 & 2,99 \\
\hline Não & 379 & 97,01 \\
\hline
\end{tabular}

A Tabela 2 apresenta a distribuição de frequências dos fatores considerados, de acordo com o resultado da aderência ao tratamento, assim como os resultados do teste de associação qui-quadrado $\left(X^{2}\right)$ e estimativas da OR bruta, com seus respectivos intervalos de $95 \%$ de confiança e valor $p$, obtidos através do ajuste de modelos logísticos univariados. Verifica-se a associação das variáveis relacionadas aos hábitos de vida individual com a adesão ou não ao tratamento. Em relação à atividade física, a não adesão foi maior entre os que realizavam exercícios menos de uma vez por semana.

Relacionado à dieta balanceada, a não adesão mostrou-se significativa naqueles que não têm preocupação com uma ingesta saudável. A variável excesso de peso mostrou-se também significativa para a não adesão, naqueles que se consideram com mais de $8 \mathrm{Kg}$ acima do peso. Constatou-se ainda, que aqueles que ingerem, em excesso, dois dos itens listados (açúcar, sal, gordura animal, alimentos puramente industrializados e frituras) tem menos chances de aderirem ao tratamento quando comparados àqueles que não ingerem nenhum item em excesso $(\mathrm{OR}=0,55)$.

Tabela 2: Análise univariada da aderência ao tratamento em função de fatores em estudo e resultado do teste de associação qui-quadrado.

\begin{tabular}{|c|c|c|c|c|c|c|}
\hline \multirow{2}{*}{ Fatores } & \multicolumn{2}{|c|}{ Aderência } & \multirow{2}{*}{$\begin{array}{c}\text { OR } \\
\text { bruta }\end{array}$} & \multirow{2}{*}{ IC 95\% } & \multirow{2}{*}{ Valor $p$} & \multirow{2}{*}{$\begin{array}{l}\text { Teste } x^{2} \\
\text { valor } p\end{array}$} \\
\hline & Não & Sim & & & & \\
\hline Vigorosamente ativo & & & & & & 0,385 \\
\hline$\leq 1$ vez por semana & 190 & 104 & 0,63 & $0,33-1,23$ & 0,175 & \\
\hline $1-2$ vezes por semana & 16 & 15 & 1,09 & $0,42-2,78$ & 0,863 & \\
\hline 3 vezes por semana & 18 & 9 & 0,58 & $0,21-1,57$ & 0,288 & \\
\hline 4 vezes por semana & 4 & 4 & 1,16 & $0,24-5,51$ & 0,850 & \\
\hline
\end{tabular}


$\geq 5$ vezes por semana

Moderadamente ativo

$\leq 1$ vez por semana

1-2 vezes por semana

3 vezes por semana

4 vezes por semana

$\geq 5$ vezes por semana

Dieta balanceada

Quase nunca

Raramente

Algumas vezes

Com relativa frequência

Quase sempre

Ingere em excesso

Quatro itens

Três itens

Dois itens

Um item

Nenhum

Intervalo para peso saudável

$>8 \mathrm{~kg}$

$8 \mathrm{~kg}$

$6 \mathrm{~kg}$

$4 \mathrm{~kg}$

$2 \mathrm{~kg}$

\section{Fuma cigarros}

$>10$ por dia

1-10 por dia

Nenhum em 6 meses

Nenhum no ano passado

Nenhum em 5 anos

Doses de álcool por semana
$22 \quad 19 \quad 1,00$

$\begin{array}{ccccc}76 & 37 & 0,65 & 0,39-1,06 & 0,086 \\ 34 & 18 & 0,70 & 0,36-1,33 & 0,287 \\ 33 & 16 & 0,64 & 0,32-1,24 & 0,198 \\ 10 & 7 & 0,93 & 0,32-2,54 & 0,889 \\ 97 & 73 & 1,00 & - & -\end{array}$

0,418

0,067

$\begin{array}{ccccc}67 & 27 & 0,49 & 0,28-0,83 & <0,01 \\ 10 & 4 & 0,49 & 0,13-1,51 & 0,238 \\ 43 & 25 & 0,71 & 0,39-1,25 & 0,237 \\ 34 & 16 & 0,57 & 0,29-1,10 & 0,099 \\ & 96 & 79 & 1,00 & -\end{array}$

0,309

$\begin{array}{ccccc}15 & 7 & 0,61 & 0,22-1,53 & 0,309 \\ 26 & 15 & 0,76 & 0,37-1,51 & 0,437 \\ 55 & 23 & 0,55 & 0,31-0,96 & 0,040 \\ 57 & 32 & 0,74 & 0,43-1,24 & 0,255 \\ 97 & 74 & 1,00 & - & -\end{array}$

$<0,01$

$114 \quad 55 \quad 0,50 \quad 0,31-0,8<0,01$

$12 \quad 5 \quad 0,43 \quad 0,13-1,23 \quad 0,133$

$17 \quad \begin{array}{lllll}14 & 0,85 & 0,38-1,87 & 0,687\end{array}$

$43 \quad 15 \quad 0,36 \quad 0,18-0,70<0,01$

$64 \quad 62 \quad 1,00$

0,091

$\begin{array}{lllll}11 & 7 & 0,96 & 0,35-2,51 & 0,941\end{array}$

$21 \quad 3 \quad 0,22 \quad 0,05-$

0,015

$\begin{array}{lllll}3 & 1 & 0,50 & 0,02-3,99 & 0,556\end{array}$

$\begin{array}{lllll}9 & 4 & 0,67 & 0,18-2,11 & 0,517\end{array}$

$206 \quad 136 \quad 1,00$

$>20$ *

$13-20$ *

$\begin{array}{ll}1 & 0 \\ 1 & 0\end{array}$

0,897 


\begin{tabular}{|c|c|c|c|c|c|c|}
\hline $11-12$ * & 1 & 0 & & & & \\
\hline $8-10$ & & 5 & 5 & 1,66 & $\begin{array}{c}0,45- \\
6,05\end{array}$ & 0,431 \\
\hline $0-7$ & 242 & 146 & 1,00 & - & - & \\
\hline Mais de 4 doses em uma ocasião & & & & & & 0,029 \\
\hline Quase diariamente * & 0 & 1 & & & & \\
\hline Com relativa frequência * & 8 & 0 & & & & \\
\hline Ocasionalmente & 35 & 13 & 0,55 & $0,27-1,05$ & 0,080 & \\
\hline Quase nunca & 29 & 16 & 0,81 & $0,41-1,54$ & 0,531 & \\
\hline Nunca & 178 & 121 & 1,00 & - & - & \\
\hline
\end{tabular}

OR: odds ratio;

IC 95\%: Intervalo de 95\% de confiança para OR;

* a estimativa da OR não foi considerada devido à baixa frequência.

Esta pesquisa explorou alguns fatores que afetam a adesão ao tratamento entre os pacientes hipertensos da cidade de Maringá. Os dados obtidos por questionários revelaram que apenas $38 \%$ da amostra adere corretamente ao tratamento anti-hipertensivo, de acordo com o TMG, resultado semelhante ao encontrado no estudo de CAVALARI (2013) que constatou que a adesão ao tratamento é baixa, estando a não adesão acima dos $50 \%$. Segundo RUZICKA (2015), na maioria dos casos a falta de adesão não é intencional, mas é um processo passivo, quer devido ao esquecimento e/ou pela incapacidade de seguir as instruções. Isso também foi encontrado no presente estudo, visto que $59,6 \%$ dos entrevistados deixaram de tomar a medicação por esquecimento. Em contraste, a não adesão intencional é um processo ativo no qual o paciente escolhe renunciar à terapia prescrita (descontinuar, pular doses ou modificá-las).

A não adesão ao tratamento é uma preocupação antiga no Brasil que, desde 1986 buscava-se soluções para reduzir as complicações e os custos com pacientes hipertensos (SOUZA \& SILVA, 1986). MEDEIROS et al. (2006) previram que entre 2005 e 2015 teríamos a morte prematura de pessoas em idade reprodutiva, por complicações da HAS, e com isso um prejuízo estimado em 40 bilhões de dólares. Além disso, a não adesão leva a altos índices de absenteísmo laboral, licenças para tratamento e aposentadorias por invalidez, o que sem dúvidas onera os cofres públicos (MEDEIROS, 2006, BARRETO, 2015). Na tentativa de melhor analisar a situação e para levantar informações acerca do agravo das doenças crônicas foi criado em 2002 pelo Ministério da Saúde o HIPERDIA, que é o sistema de acompanhamento e cadastramento de hipertensos e diabéticos preconizado pelo Plano Nacional de Reorganização a Atenção à Hipertensão e Diabetes (BOING, 2007), programa do qual toda amostra fazia parte. Mesmo com todo esse aparato, o custo anual estimado para o tratamento da hipertensão arterial no sistema público de saúde brasileiro gira em torno US\$ 398,9 milhões e representa $1,43 \%$ dos gastos totais do SUS (DIB, 2010). O aumento do número de idosos, somado aos maus hábitos alimentares e sedentarismo são os principais responsáveis pela elevação dos gastos com 
doenças cardiovasculares (LADEIRA, 2016), o que se acredita que possa ser mudado com ações realizadas pela atenção básica, de forma a reduzir a não adesão aos tratamentos indicados (CUNHA et al., 2012).

A adesão ao tratamento envolve o uso de medicamentos bem como mudanças no comportamento, sendo esse um fenômeno complexo influenciado por vários fatores relacionados ao estilo de vida. Já foram discutidas e constatadas a relação da hipertensão e complicações relacionadas à obesidade, inatividade física, alta ingesta de sal, tabagismo e fatores socioeconômicos (VACINI-CAMPANHARO et al., 2015).

Notou-se que os pacientes que não tem hábitos de dieta balanceada apresentam cerca da metade das chances de aderir ao tratamento quando comparados àqueles que se alimentam de modo balanceado quase sempre. Da mesma forma, aqueles que ingerem dois dos itens listados (açúcar, sal, gordura animal, alimentos puramente industrializados e frituras) em excesso, apresentam menores chances de aderência quando comparados àqueles que não ingerem nenhum item em excesso. Como apresentado na tabela 2, aqueles que quase nunca se preocupam com a alimentação, significativamente, não aderem ao tratamento. Sabe-se que a adesão às recomendações dietéticas são ainda menores que a adesão ao uso de drogas anti-hipertensivas. GALLETTI et al., (2014) evidenciaram que apenas 10 e 19\% dos homens e mulheres com HAS, respectivamente, foram aderentes ao baixo teor de sódio na dieta, e apenas $5-8 \%$ destes pacientes apresentavam um adequado consumo de potássio. $O$ aumento de doenças como hipertensão arterial sistêmica, obesidade, doença coronariana, diabetes e câncer já foi atribuído ao padrão alimentar moderno, em que houve aumento do consumo de gordura, açúcar e carne, com queda na ingestão de fibras. Devido à isso há um acréscimo nos níveis pressóricos, o que aumenta o risco de eventos ateroscleróticos e consequentemente a morbimortalidade (BURKITT, 1993).

$\mathrm{Na}$ tentativa de que a dieta não fosse mais um fator preditor para a HAS criou-se a DASH (Dietary Approaches to Stop Hypertension) que enfatiza o consumo de frutas, hortaliças e laticínios com baixo teor de gordura; inclui a ingestão de cereais integrais, frango, peixe e frutas oleaginosas; preconiza a redução da ingestão de carne vermelha, doces e bebidas com açúcar. A adoção desse padrão alimentar já demonstrou reduzir significativamente a pressão arterial (SOCIEDADE BRASILEIRA DE CARDIOLOGIA, 2016). Para que os pacientes adotem um novo estilo de vida é necessário o envolvimento de toda equipe multidisciplinar, na tentativa de auxiliar o paciente de forma individualizada, objetivando o não abandono do tratamento. Já foi demonstrado que a atuação da Atenção primária varia entre $10 \%$ e $57 \%$ nesse controle pressórico, e que a atuação em equipe consegue obter um melhor controle da pressão arterial para os hipertensos (PINHO, 2013).

Estudos apontam que grande parte dos pacientes não aderentes são classificados pelo IMC, em sobrepeso ou obesidade (JESUS, 2008), e já foi comprovada a redução dos níveis pressóricos quando da adoção de uma dieta adequada (SOLTANI, 2017). Analisando essa variável observou-se que para aqueles que disseram estar acima de $4 \mathrm{~kg}$, e também aqueles que tem mais de 8 $\mathrm{kg}$ acima do peso, as chances de adesão são menores do que para os que estão 
apenas $2 \mathrm{~kg}$ acima do peso considerado ideal. Verificou-se ainda, que os indivíduos que fumam de 1 a 10 cigarros são menos propensos a aderir ao tratamento anti-hipertensivo comparados aos que não fumaram nos últimos 5 anos, o que também foi confirmado em analises anteriores, as quais demonstraram que nos indivíduos fumantes e que fazem ingesta de álcool, a não adesão ao tratamento farmacológico é de cerca de 77\% (DEMONER, 2012).

Quando comparada uma mesma variável, de forma geral, os pacientes não aderem de forma satisfatória ao tratamento, conforme observado nesta pesquisa. Isto nos leva a inferir que até mesmo os indivíduos que possuem estilo de vida mais saudável encontram dificuldades para aderir ao tratamento anti-hipertensivo, necessitando de maior atenção por parte dos profissionais da saúde. Isso também foi evidenciado por LEE (2013) que demonstrou que mesmo o paciente com autopercepção de "boa saúde", pode apresentar limitações na adesão ao regime terapêutico, influenciado pela ausência de sintomas da doença que conduz as pessoas a terem uma percepção alterada sobre a doença, o que reforça a necessidade do comprometimento da Atenção primária para a manutenção do tratamento nesses indivíduos. O conhecimento sobre a doença e o seu tratamento podem facilitar a adesão do paciente à terapia proposta, já que sua ausência pode gerar, ansiedade, insegurança, pouco importância ao tratamento, esquecimento e estresse (FERREIRA, 2014).

\section{CONCLUSÃO}

A investigação constatou a prevalência elevada de não adesão à terapia anti-hipertensiva dos pacientes cadastrados no sistema HIPERDIA de MaringáPR. Dentre os pacientes com alimentação inadequada, que mantinham hábitos de fumar, ingerir bebidas alcoólicas e/ou sedentários foi evidenciada uma forte tendência a não adesão. No entanto, mesmo que alguns indivíduos busquem um estilo de vida mais saudável, esses também não aderem significativamente ao tratamento. Cabe dizer que a mudança de hábitos e de estilo de vida dos hipertensos deve ser enfatizada, pois faz parte da assistência terapêutica, já que a adoção de um estilo de vida saudável e prática de atividade física contribuem significativamente para a redução da pressão arterial, além de auxiliar na diminuição ou manutenção do peso e diminuir os riscos cardiovasculares. Dessa forma, torna-se fundamental a ênfase nas condutas de tratamento não medicamentoso, especialmente quanto à reeducação alimentar, atividades físicas, cessação do hábito tabágico e ingesta de álcool, tarefas essas que ficariam a cargo da atenção primária e que possivelmente reduziria os riscos de complicações e consequente oneração do sistema público. A investigação possibilita ainda, nortear intervenções a essa clientela, além de subsidiar novos estudos para a implementação de novas estratégias nesse âmbito. O presente estudo limitou-se a análise dos dados quantitativos do instrumento de MoriskyGreen.

\section{AGRADECIMENTOS}

Ao Instituto Cesumar de Ciência, Tecnologia e Inovação - ICETI e Conselho Nacional de Desenvolvimento Científico e Tecnológico - CNPq pela concessão de bolsas de auxílio pesquisa. 


\section{REFERÊNCIAS}

BARRETO, M.S.; CREMONESELL, I.Z.; JANEIROL, V.; MATSUDA, L.M.; MARCON, S.S. Prevalência de não adesão à farmacoterapia anti-hipertensiva e fatores associados. Revista Brasileira de Enfermagem, v. 68, n. 1, p. 60-67, 2015. Disponível em:< http://dx.doi.org/10.1590/0034-7167.2015680109p>. Doi:10.1590/0034-7167.2015680109p.

BEZERRA, A.S.M.; LOPES, J.L., BARROS, A.L.B.L. Adesão de pacientes hipertensos ao tratamento medicamentoso. Revista Brasileira de Enfermagem. v. 67 , n. 4, p. 550-555, 2014. Disponível 8" http://dx.doi.org/10.1590/00347167.2014670408. Doi: 10.1590/0034-7167.2014670408.

BOING, AC; BOING, AF. Hipertensão Arterial sistêmica: o que nos dizem os sistemas brasileiros de cadastramentos e informações em saúde. Revista Brasileira de Hipertensão, v.14, n.2, p.84-88, 2007. Disponível ef" http://departamentos.cardiol.br/dha/revista/14-2/06-ipertensao.pdf>.

BORGES, JWP; MOREIRA, TMM; RODRIGUES, MTP; OLIVEIRA, CJ. Utilização de questionários validados para mensurar a adesão ao tratamento da hipertensão arterial: uma revisão integrativa. Revista Escola Enfermagem USP, v. 46, n. 2, p. 487-494, $2012 . \quad$ Disponível em0" http://www.scielo.br/pdf/reeusp/v46n2/a30v46n2.pdf>.Doi:10.1590/S008062342012000200030 .

BURKITT, D.P. Some diseases characteristic of modern westwm civilization. Britsh Medical Journal. v. 1, n. 5848, p.274-278, 1993.

CAVALARI, E.; NOGUEIRA, M.S.; CESARINO, C.B.; MARTIN, J.F.V. Adesão ao tratamento: estudo entre portadores de hipertensão arterial em seguimento ambulatorial. Revista de Enfermagem UERJ. v. 20, n. 1, p. 67-72, 2012. Disponível ef" http://www.facenf.uerj.br/v20n1/v20n1a12.pdf.

CESARINO, CB; CIPULLO, JP; MARTIN, JFV; CIORLIA, LA; GODOY, MRP; CORDEIRO, JA, et al. Prevalência e fatores sociodemográficos em hipertensos de São José do Rio Preto. Arquivo Brasileiro de Cardiologia, v.91, n.1, p.31-35, 2008. Disponível e5" http://www.scielo.br/scielo.php?script=sci_arttext\&pid=S0066782X2008001300005. doi.org/10.1590/S0066-782X2008001300005.

CUNHA, P.R.M.S. Et al. Prevalência e causas de não adesão ao tratamento antihipertensivos de idosos na Atenção Básica. Revista Pesquisa Saúde, v. 13, n. 3 , p. 11-16, 2012.

DEMONER, MS; RAMOS, ERP; PEREIRA, ER. Factors associated with adherence to antihypertensive treatment in a primary care unit. Acta Paulista de Enfermagem, v. 25, n. 1, p. 27-34, 2012. Disponível em5" 
http://www.scielo.br/scielo.php?script=sci_arttext\&pid=S0103-

21002012000800005>. Doi:10.1590/S0103-21002012000800005.

DIB, MW; RIERA, R; FERRAZ, MB. Estimated annual cost of arterial hypertension treatment in Brazil. Revista Panamamericana de Salud Publica/Pan American Journal of Public Health, v. 27, n. 2, p. 125-131, 2010. Disponível ef" http://www.scielosp.org/pdf/rpsp/v27n2/a06v27n2.pdf. Doi: 10.1590/S102049892010000200006

FERREIRA, R.A.; BARRETO, S.M.; GIATTI, L. Hipertensão arterial referida e utilização de medicamentos de uso contínuo no Brasil; um estudo de base populacional. Caderno de Saúde Pública. v. 30, n. 4, p. 815-826, 2014. DOI: 10.1590/0102-311X00160512

GALLETTI, F; AGABITI-ROSEI, E; BERNINI, G; et al. Excess dietary sodium and inadequate potassium intake by hypertensive patients in Italy: results of the MINISAL-SIIA study program. Journal of Hypertension, v. 32, p. 48-56, 2014. Disponível em9" https://www.ncbi.nlm.nih.gov/pubmed/24275839>. Doi: 10.1097/HJH.0000000000000010.

JESUS, ES; AUGUSTO, MA; GUSMÃO, J; MION JUNIOR, D; ORTEGA, K; PIERIN, AM. Profile of hypertensive patients: biosocial characteristics, knowledge, and treatment compliance. Acta Paulista de Enfermagem, v. 21, n. 1, p. 59-65, 2008. Disponível emf" http://www.scielo.br/pdf/ape/v21n1/08.pdf>. Doi:10.1590/S0103-21002008000100009.

$\mathrm{JOHO}, \mathrm{AA}$. Factor affecting treatmento compliance among Hypertension patients in three district hospitals - Dar es Salaam. 2012. Dissertation [Degree of Master of Science Nursing (Critical Care \& Trauma)] - Muhimbili University of Health and Allied Sciences; 2012.

KEARNEY, P; WHELTON, M.; REYNOLDS, K.; MUNTNER, P.; WHELTON, P.K.; HE, J. Global burden of hypertension: analysis of worldwide data. The Lancet, v.365, p.217-23, 2005. Disponível em:<https://www.ncbi.nlm.nih.gov/pubmed/1565260>. D1" 10.1016/S01406736(05)17741-1.

KRONISH, IM; WOODWARD, M; SERGIE, Z; OGEDEGBE, G; FALZON, L; MANN, DM. Metaanalysis: impact of drug class on adherence to antihypertensives. Circulation, v. 123, p. 1611-1621, 2011. Disponível e0" https://www.ncbi.nlm.nih.gov/pubmed/21464050>. Doi:10.1161/CIRCULATIONAH A.110.983874.

LADEIRA, R.C.; ERTHAL JÚNIOR, M.; DA HORA, H.R.M. Métodos de escolha de medicamentos anti-hipertensivos por gestores da área da saúde. Acta Biomedica Brasileira. v. 7, n. 1, p. 48-63, 2016. Disponível em8" http://dx.doi.org/10.18571/acbm.098>. Doi: 10.18571/acbm.098. 
LEE, G.K.Y.; WANG, H.H.X.; LIU, K.Q.L., CHEUNG, Y.U.; MORISKY, D.E.; WONG, M.C.S. Determinants of Medication Adherence to Antihypertensive Medications among a Chinese Population Using Morisky Medication Adherence Scale. PLOS ONE, v. 8, n.4, p. 1-7, 2013. DO5" 10.1371/journal.pone.0062775.

LIMA, D.B.S.; MOREIRA, T.M.M., BORGES, J.X.P.; RODRIGUES, M.T.P. Association between treatment compliance and diferente types os cardiovascular complications in hypertension patients. Texto Contexto de Enfermermagem, v. 25, n. 3, eo5o6oo15, 2016. Disponível em5" http://dx.doi.org/10.1590/010407072016000560015>. DOI: 10.1590/0104-07072016000560015.

MEDEIROS, S.B.; Et al. Obesidade, tabagismo e hipertensão - Fardos financeiros do sistema de saúde. Rio de Janeiro, Federação da Indústria do Estado do Rio de Janeiro Serviço Social da Indústria - SESI - Diretoria de Saúde, 40p., 2006.

MORISKY, DE; GREEN, LW; LEVINE, DM. Concurrent and predictive validity of self-reported measure of medication compliance. Medical Care, v. 24, p. 67-74, 1986. Disponível em0" https://www.ncbi.nlm.nih.gov/pubmed/3945130>.DOI:10.1097/00005650198601000-00007.

MORISKY, DE; ANG, A; KROUSEL-WOOD, M; WARD, HJ. Predictive validity of a medication adherence measure in an outpatient setting. Jounal of Clinic Hypertension (Greenwich, Conn), v.10, n.5, p. 348-354, 2008. Disponível e3" https://www.ncbi.nlm.nih.gov/pubmed/18453793>.DOI:10.1111/j.1751-

7176.2008.07572.x.

PINHO, N.A.; PIERIN, A.M. Hypertension control in brazilian publications. Arquivo Brasileiro de Cardiologia. v. 101, n. 3, p. 65-73, 2013. Disponível em0" http://www.scielo.br/scielo.php?script=sci_arttext\&pid=S0066-

782X2013002900020> DOI: 10.5935/abc.20130173.

RABBIA, F.; FULCHERI, C.; DI MONACO, S.; COVELLA, M.; PAPPACCOGLI, M.; VEGLIO, F. Adherence to antihypertensive therapy and therapeutic dosage of antihypertensive drugs. High Blood Pressure \& Cardiovascular Prevention, Disponível em:Z" https://www.infona.pl/resource/bwmeta1.element.springer-doi10_1007-S40292-016-0158-Z>. DOI 10.1007/s40292-016-0158-Z. Published online 09 May 2016.

RAMOS, L.L. Adesão ao tratamento anti-hipertensivo na Estratégia Saúde da família de Sobrália, Minas Gerais. 2014. 36p. Trabalho de conclusão de curso (Especialização em Atenção básica em Saúde da Família) - Universidade Federal de Minas Gerais, Minas Gerais, 2014.

ROSÁRIO, TM; SCALA, LCNS; FRANÇA, GVA; PEREIRA, MRG; JARDIM, PCBV. Prevalência, controle e tratamento da hipertensão arterial sistêmica em Nobres, 
MT. Arquivo Brasileiro Cardiologia, v. 93, n. 6, p. 672-678, 2009. Disponível ef" http://www.scielo.br/pdf/abc/v93n6/18.pdf. $782 \times 2009001200018$.

doi.org/10.1590/S0066-

RUZICKA, M.; HIREMATH, S. Can Drugs Work in Patients Who Do Not Take Them? The Problem of Non-adherence in Resistant Hypertension. Current Hypertension Reports, 2015. Disponível https://www.ncbi.nlm.nih.gov/pubmed/26164465. DOI 10.1007/s11906-015-0579-4.

SANTOS, M.V.R.; OLIVEIRA, D.C.; ARRAES, L.B.; OLIVEIRA, D.A.G.C.; MEDEIROS, L.; NOVAES, M.A. Adesão ao tratamento anti-hipertensivo: conceitos, aferição e estratégias inovadoras de abordagem. Revista Brasileira de Clínica Médica, v. 11, n. 1, p. 55-61, 2013. Disponível emf" http://files.bvs.br/upload/S/1679-1010/2013/v11n1/a3390.pdf>.

Sociedade Brasileira de Cardiologia. VII Diretrizes Brasileira de Hipertensão Arterial. Arquivos Brasileiros de Cardiologia, v. 107, n. 3, p. 30-34, 2016.

SOLTANI, S.; CHITSAZI, M.J.; ABARGOUEI, A.S. The effect of Dietary Approaches to Stop Hypertension (DASH) on serum inflammatory markers: a systematic review and meta-analysis of randomized trials, Clinical Nutrition (2017), doi: 10.1016/j.clnu.2017.02.018.

SOUZA E SILVA, NA; AGUIAR, G.R.; NOGUEIRA, A.R.; DUARTE, M.M.T.; ALVES, R.H.F. Importância clínica dos custos hospitalares em pacientes com hipertensão arterial em tratamento num hospital universitário, Rio de Janeiro, Brasil. Revista de Saúde Pública, v. 20, p. 293-302, 1986. Disponível ef" http://www.scielo.br/pdf/rsp/v20n4/05.pdf. 89101986000400005.

TALAEI, M.; SADEGHI, M.; MOHAMMADIFARD, N.; OVEISGHARAN, S.; SARRAFZADEGAN, N. Incident hypertension and its predictors: the Isfahan Cohort Study. Journal Hyertension, v.32 n.1 p.30-38, 2014. Disponível e9" https://www.ncbi.nlm.nih.gov/pubmed/24048009. doi: 10.1097/HJH.0b013e32836591d4.

VACINI-CAMPANHARO, C.R.; OLIVEIRA, G.N.; ANDRADE, T.F.L.; OKUNO, M.F.P.; LOPES, M.C.B.T.; BATISTA, R.E.A. Hipertensão Arterial Sistêmica no serviço de emergência: adesão medicamentosa e conhecimento da doença. Revista latino-americana enfermagem, v. 23, n. 6, p. 1149-1156, 2015. Disponível emf" http://www.scielo.br/pdf/rlae/v23n6/pt_0104-1169-rlae-23-06-01149.pdf>. Doi: 10.1590/0104-1169.0513.2660.

VARLETA, P; Et al. Prevalencia y determinantes de adherencia a terapia antihipertensiva en pacientes de la Región Metropolitana. Revista Medica de Chile, v. 143, p. 569-576, 2015. Disponível emf" 
http://www.scielo.cl/pdf/rmc/v143n5/art03.pdf>.

Doi:10.4067/S003498872015000500003.

WORLD HEALTH ORGANIZATION. Global Atlas on Cardiovascular Disease Prevention and Control. World Health Organization; 2011. Disponível em/" http://www.who.int/cardiovascular_diseases/publications/atlas_cvd/en/>. ISBN: 978 9241564373.

YGUSHI, N.Y. Adesão ao tratamento e controle da pressão arterial: considerações a partir de revisões bibliográficas. 2013. 48p. Trabalho de conclusão de curso (Especialização em Atenção básica em saúde da Família) Universidade Federal de Minas Gerais, Minas Gerais, 2013. 